\title{
Novel expression of microRNAs in serum samples of Iraqi breast cancer women
}

Zaynab Saad ${ }^{1}$; Muhammed Arif ${ }^{2}$; Nahi Yassen ${ }^{1}$; Hameed Jasim³ ${ }^{3}$ : Majed Jelawe ${ }^{3}$; James Brown $^{2}$

\begin{abstract}
Although a lot of hard work against cancer to reduces its spread but it still continues to kill with abandon. The need for a biomarker for cancer early detection becomes the most mind concentrated scientists. MicroRNAs the tiny non coding RNA molecules opened new path for the scientists to determine the cancer in its early stages. Expression of microRNAs profiles has been investigated to be involved in cancer development. Here we determined the expression of microRNAs in serum of Iraqi healthy volunteers and other women diagnosed with breast cancer. MicroRNAs expression has been determined by using real time qPCR and delta method has been used. Four of thirteen microRNAs were shown to be expressed in serum of Iraqi breast cancer women. Let-7a and miR-21 were shown to be significantly over expressed in serum of breast cancer compared with healthy serum volunteers $(P=$ 0.022 and 0.026 ) respectively. While miR-26b and miR-429 found to be significantly down expressed in serum of breast cancer women $(P=0.0034$ and 0.031$)$ respectively. The result concluded that these expressed microRNAs in serum of breast cancer women could be used as a first indicator of breast cancer occurrence.
\end{abstract}

Keywords: microRNA; Breast cancer; Real Time PCR; Reverse Transcription

*Corresponding Author: Zaynab Saad: Zaynab_saad@yahoo.com

${ }^{1}$ Al Mustansiriya University/ Baghdad/Iraq

${ }^{2}$ Aston University / Birmingham/ UK

${ }^{3}$ Al- Nahrain University/ Baghdad/ Iraq

Received 18 March 2014; accepted 22 April 2014

Copyright $\odot 2014$ ZS. et al. This is article distributed under the terms of the Creative Commons Attribution License (http://creativecommons.org/licenses/by/2.0), which permits unrestricted use, distribution, and reproduction in any medium, provided the original work is properly cited. 


\section{Introduction}

Although a lot of hard work against cancer but it still continues to kill with abandon [1]. The increase incidence of breast cancer in Iraqi women has been started after the Gulf war 1990 and the second war 2003[2]. The need for specific and sensitive diagnostic biomarkers for early detection and monitor of breast cancer has let the scientists to see; for a marker that predict the breast cancer in an early stages [3]. Such biomarker must be sensitive, non-invasive and not affected by environmental conditions and may facilitate in early detection and diagnose of breast cancer [4]. MicroRNA which are small in size about $23 \mathrm{nt}$, non-coding RNA molecules has been shown to be sequences conserved among plant, animal, human and even insect [5].

MicroRNA found to regulate gene expression and involved in biological processes including, development, differentiation, proliferation and apoptosis [6]. The function of microRNA could be summarized by its binding with the 3'-UTR of the target mRNA gene, and by this binding microRNA will target and control the expression of that gene [7]. So that microRNA expression profiling may provide a more accurate classification of cancer subtypes, diagnosis and prognosis. Micro RNA has been shown to be present in human biological fluids (serum/plasma, saliva, tears and urine) that will facilitate the diagnosis of disease especially cancer [8]. Current study was aimed to determine the expression of microRNAs in serum samples collected from Iraqi healthy women and breast cancer diagnosed women.

\section{Materials and methods}

\section{Serum sample collection}

All women volunteer that participate in current study were interviewed and questioned and all information was recorded. Whole blood sample was collected in a tube containing gel for serum separation, from healthy women and women diagnosed with breast cancer. Blood samples left at room temperature for $30 \mathrm{~min}$. to allow serum separation then spun at $1500 \mathrm{~g}$ for $10 \mathrm{~min}$. The serum was transferred to a new eppendorf tubes and frozen at $-80 \mathrm{C}$ until microRNA extraction. 


\section{MicroRNA extraction}

The circulating microRNAs were extracted from serum samples using microRNA extraction kit (Norgen Biotek, Canada) using 200 $\mu 1$ of serum aliquots. All serum samples were thawed completely, and then $200 \mu$ of serum mixed with denaturation buffer in a procedure described in the kit protocol. The microRNA at the end was eluted by $50-100 \mu \mathrm{l}$ of nuclease free water. The microRNA concentration was measured using NanoDrop-1000 spectrophotometer (Nano Drop Technologies, USA).

\section{Reverse Transcription}

The extracted microRNA was reversed transcribed using TaqMan microRNA reverse transcription kit (ApplidBiosystems, USA) according to the manufactures protocol. Then $5 \mu \mathrm{l}$ of microRNA was reversed transcribed in a $15 \mu \mathrm{l}$ reaction volume for each assay.

\section{Real Time PCR}

TaqMan microRNA probes were used (miR-21, let-7a, miR-222, miR-26b, miR-27a, miR-15b, miR-34a, miR-34b, miR-205, miR-218, miR-378, miR-429 and miR-191 was used as an endogenous control) were used to quantify microRNA in real time PCR assays according to manufactures protocol. Real time PCR assays were performed in a $20 \mu$ reaction volume on a Stratagen $3000 \mathrm{p}$ real time system.

\section{Statistical analysis}

Student $t$ test $\mathrm{p}$ value was calculated using GraphPad Prism 6.

\section{Results}

The microRNAs were isolated from serum of six Iraqi healthy and fifteen breast cancer diagnosed women and the expressions of these microRNAs have been determined by q-RT-PCR. We selected four miRNAs that statistically significant. We found that let-7a and miR-21 expressions was up-regulated in breast cancer patients compared with the healthy controls $(P=0.022$ and 0.026$)$ respectively as indicated in figure $(1, \mathrm{~A}$ and $\mathrm{B}$ respectively; $P<0.05)$ while miR-26b and miR-429 expression were down- regulated in breast cancer patients compared with the healthy controls 
$(P=0.0034$ and 0.031$)$ respectively as indicated in figure $(1, C$ and $D$ respectively; $P<0.05)$.

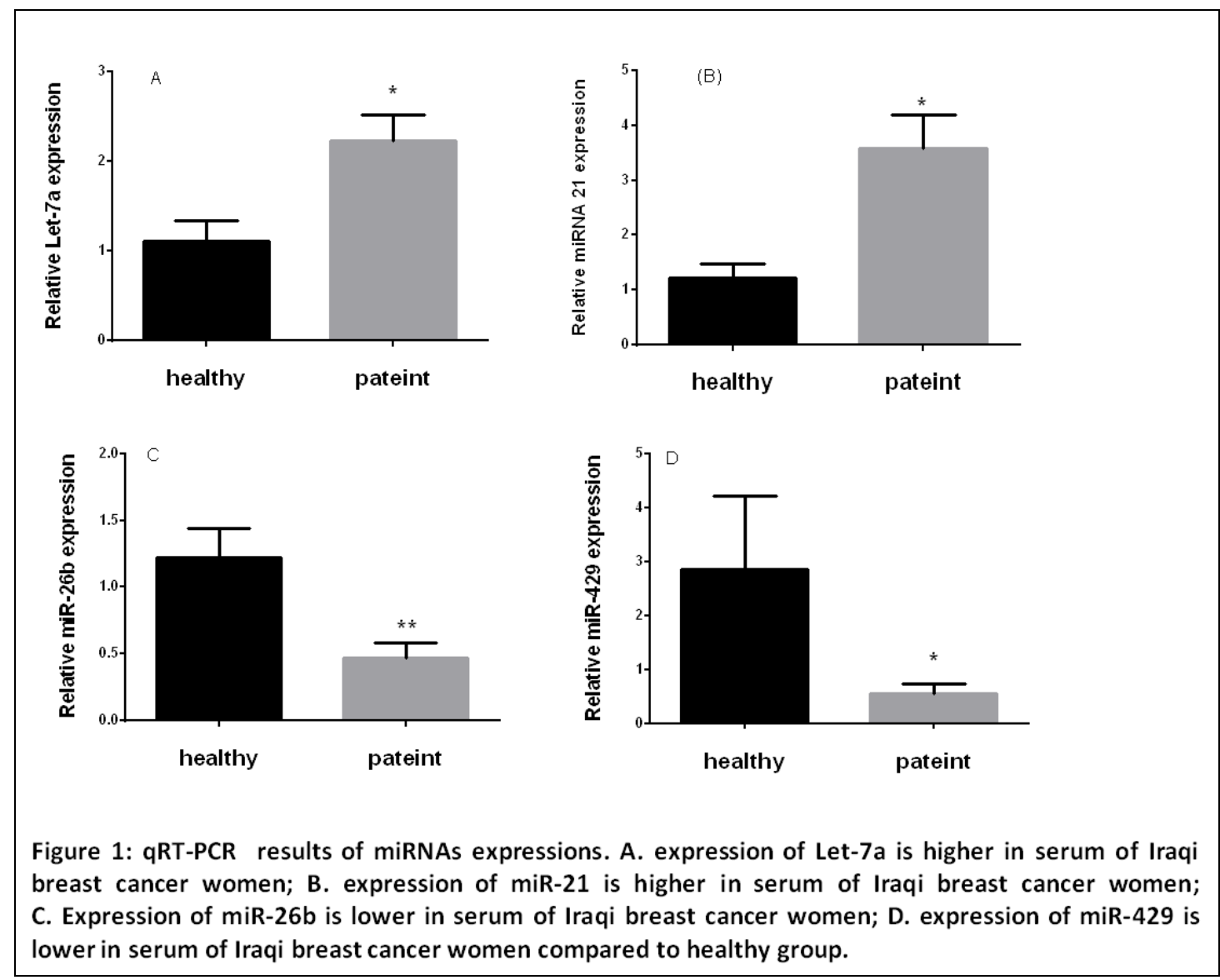

\section{Discussion}

Breast cancer represent the second cancer leading to death in women, is expected to be $26 \%$ of new cancer diagnosis [9]. The need for specific biomarker for an early detection of cancer represents the aim of all scientist work right now. The discovery of microRNA in 1993 by lee and coworker opened new sights about these small molecules [10], after that in 2008 they found that these tiny RNA molecules are circulating of cancer patients followed by a huge number of studies determining microRNA importance in early detection of cancers [11]. The detection of some novel microRNAs increase the studies attentions. Some of the microRNAs that used in this study were detected before and some novels have not been detected before. MiRNAs are very stable in blood plasma and serum since protected from RNases [12]. Thus, it makes miRNA levels tested for clinical diagnosis as tumor markers and biomarkers. 
Chim et al., 2008 have suggested two hypotheses for the source of miRNAs on circulating blood samples; it may be due to a result of tumor cell death and lyses or release by tumor cells into the extracellular microenvironment of blood vessels [13]. In this study we analyzed the expression of thirteen microRNAs in serum from fifteen breast cancer women patients and six healthy women as controls. Our study showed a significant high expression of let-7a and miR-21 in serum of breast cancer patients as mentioned in previous studies of Heneghan et al., 2010 and Mar- Aguilar et al., 2013 $[14,15]$.

Let-7a found to be involved in cell proliferation and has role in cancer metastasis in various tumors, especially breast cancer [16], our result indicate that let-7a tends to be up regulated in serum of breast cancer patients. MiR-21 found to participate in regulating the expression of multiple tumor suppressor genes, and played an important role in the development of colon cancer, lung cancer, stomach cancer and breast cancer [17] like here in current study, miR-21 was up regulated in serum of breast cancer patients. MiR-26 has shown to be a key regulator in carcinogenesis and tumor progression by acting either as oncogenes or tumor suppressor genes in various cancers [18]. MiR-26 is consistently down-regulated in a wide range of malignant tumors, such as hepatocellular carcinoma, lung cancer and breast cancer [19]. Breast cancer women have down expressed miR-26b in their serums. The miRNA-200 cluster has been shown to regulate the epithelial-mesenchymal plasticity that may be crucial at different stages of metastasis [20].

It has been suggested that the miR-200 family is a powerful marker and an essential regulatory factor of the cancer cell epithelial phenotype [21]. The miR-200 family of miRNAs consists of five members: miR-200a, 200b and 429 [22]. The serum level of miR-429 in breast cancer women was down expressed. The serum levels of miR-200a, miR-200b, miR-200c, miR-429, miR-205 and miR-192, and urinary miR-200a, miR-200c, miR-141, miR-429 and miR-192 of SLE patients were lower than those of controls [23]. The results indicated that there is a possibility of detection the changes in the level of microRNAs expression as useful biomarkers for the detection and early diagnosis of breast cancer.

In Conclusion; the significant over expression of let-7a and miR-21 in circulation of Iraqi breast cancer women and down expression of miR-26b and miR-429 in the same group have been detected and suggested to be new microRNAs in early detection of breast cancer. 


\section{Competing interests}

The authors have declared that no competing interests.

\section{Authors' Contributions}

ZS, HM, NY, MJ designed the study. ZS, MA carried out the experiments. ZS drafted the manuscript. MA, JB participated in the experiments and data analysis. All authors read and approved the final manuscripts.

\section{Funding}

This study was supported and offered by Republic of Iraq / Ministry of Higher Education and Scientific Research.

\section{References}

1. Jain S. Malignant: How cancer become us. University of California Press 2013.Iraqi Cancer Board. Results of Iraqi Cancer Registry 2004-2005. Ministry of Health. Baghdad-Iraq, 2010.

2. Alex A. Novel anticancer agents: strategies for discovery and clinical testing. Chapter 10: Surrogate end points and bomarkers for early trails of novel anticancer agents. Academic Pr./ USA, 2006.

3. Rosenfeld N, Aharonov R, Meiri E. MicroRNAs accurately identify cancer tissue origin. Nat. Biotechnol 2008; 26:462-469.

4. Ambros V. The functions of animal microRNAs. Nature 2004; 431: 350-355.

5. Cimmino A, Calin G, Fabbri M, Iorio M, Ferracin M, Shimizu M. MiRNA-15 and miRNA-16 induce apoptosis by targeting BCL2. Proc. Natl. Acad. Sci. USA 2005; 102: 13944-13949.

6. Kim V, Nam J. Genomics of miRNA. TRENDS in Genetics 2006; 22: 165.

7. Cortez M, Bueso-Ramos C, Ferdin J, Sood A, Calin G. MicroRNAs in body fluidsthe mix of hormones and biomarkers. Rat. Rev. Clin. Oncol 2011; 8:467-477. 
8. Kantarjian H, Wolff R, Koller C. The MD Anderson Manual of Medical Oncology. $2^{\text {nd }}$ ed. McGrawHill Medical Inc. US, 2011.

9. Lee R, Feinbaum R, Ambros V. The C. elegans heterochronic gene lin-4 encodes small RNAs with antisense complemetarity to lin-14. Cell 1993;75: 843-854.

10.Mitchell P, Parkin R, Kroh E, Fritz B, Wyman S. Circulating miRNAs as stable blood- based markers for cancer detection. Proc. Natl. Acad. Sci. USA 2008; 105: 10513-10518.

11.Krol J, Loedige I, Filipowicz W. The wide separed regulation of microRNA biogenesis, function and decay. Nature Rev. Genet 2010; 11:579-610.

12.Chim S, Shing T, Hung E, Leung T. Detection and charachterization of placental microRNA in maternal plasma. Clin. Chem 2008; 54:482-490.

13.Heneghan H, Miller N, Kelly R, Newell J. Systemic miR-195 diffrentiate breast cancer from other malignancies and is a potential biomarker for detecting noninvasive and early stage disease. Oncologist 2010; 15: 673-682.

14.Marc E, Jean-Pierre D, Thierry C. Breast cancer incidence and hormone replacement therapy: Results from mission study, prospective phase. J. Gyne. Endo 2007; 23: 391-397.

15.Zhang $\mathrm{H}$, Li Y, Lai M. The microRNA network and tumor metastasis. Oncogene 2010; 29: 937-948.

16.Gao J, Zhang Q, Guo L, Xu J. Clinical Significance of serum miR-21 in breast cancer compared with CA15-3 and CEA. Chin. J. Cancer Res 2013; 25:743-748.

17.Li J, Kong X, Zhang J, Luo Q, Li X. MiRNA-26b inhibits proliferation by targeting PTGS2 in breast cancer. Ca. Cell Int 203;13:7.

18.Gao J, Liu Q. The role of miR-26 in tumors and normal tissues (review). Oncol. Lett 2011; 2:1019-1023.

19.Burk V, Schubert J, Wellner V, Schmalhofer O, Brabletz T. A reciprocal repression between ZEB1 and members of the miR-200 family promotes EMT and invasion in cancer cells. EMBO Rep 2008; 9:582-589. 
20.Park S, Gaur A, Lengyel E, Peter M. The miR-200 family determines the epithelial phenotype of cancer cells by targeting the E-cadherin repressors ZEB1and ZEB2. Genes Dev 2008;22:894-907.

21.Hashimoto Y, Akiyama Y, Yuasa Y. Multiple- to multiple relation ships between miRNAs and targets genes in gastric cancer. PLoS One 2013;8: e62589.

22.Wang G, Tam L, Li E. Serum and Urinary free miRNA levels in pateints with systemic lupus erythematosus. Lupus 2011; 20:493-500. 